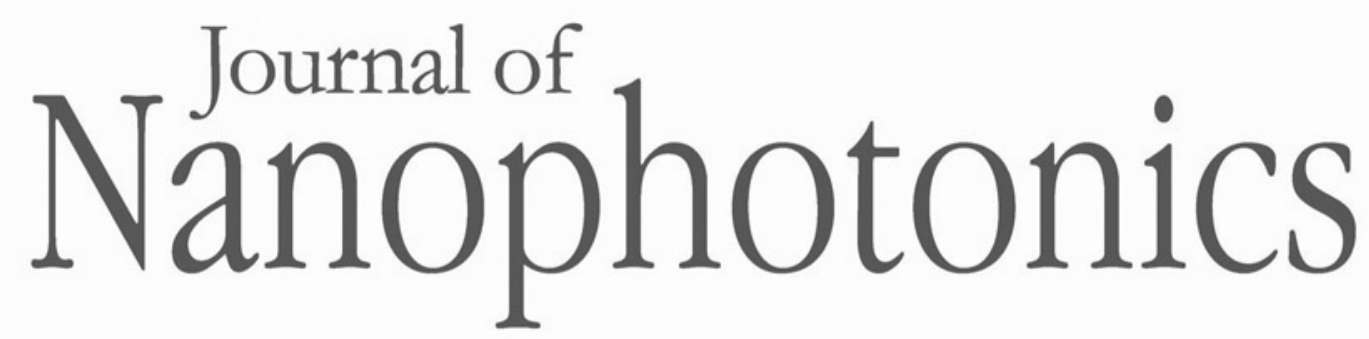

\title{
Optical fluorescent spider silk electrospun nanofibers with embedded cerium oxide nanoparticles
}

\author{
Ishac Kandas \\ Nader Shehata \\ Ibrahim Hassounah \\ Patrik Sobolčiak \\ Igor Krupa \\ Randolph Lewis
}




\title{
Optical fluorescent spider silk electrospun nanofibers with embedded cerium oxide nanoparticles
}

\author{
Ishac Kandas, ${ }^{a, b, c, d}$ 网 Nader Shehata, ${ }^{\text {a,b,c,d }}$ Ibrahim Hassounah, ${ }^{d}$ \\ Patrik Sobolčiak, ${ }^{\mathrm{e}}$ Igor Krupa, ${ }^{\mathrm{f}}$ and Randolph Lewis ${ }^{\mathrm{d}}$ \\ ${ }^{a}$ Alexandria University, Department of Engineering Mathematics and Physics, \\ Faculty of Engineering, Alexandria, Egypt \\ ${ }^{\mathrm{b}}$ Alexandria University, Center of Smart Nanotechnology and Photonics, \\ SmartCI Research Center, Alexandria, Egypt \\ ${ }^{\mathrm{c}}$ Kuwait College of Science and Technology, Doha, Kuwait \\ ${ }^{\mathrm{d} U t a h}$ State University, Department of Biology, Logan, Utah, United States \\ ${ }^{\mathrm{e}}$ Qatar University, Center of Advanced Materials, Doha, Qatar \\ ${ }^{f}$ Qatar University, QAPCO Polymer Chair, Center for Advanced Materials, Doha, Qatar
}

\begin{abstract}
The work demonstrates an electrospun nanocomposite of recombinant spider silk protein ( $\mathrm{rSSp}$ ) nanofibers with embedded cerium oxide (ceria) nanoparticles. RSSP (MaSp1) has been produced, extracted from goat milk, and fabricated into nanofibers using an electrospinning process. The resulting electrospun nanofibers have a mean diameter of $\sim 50 \mathrm{~nm}$. Furthermore, ceria nanoparticles of mean diameter $<10 \mathrm{~nm}$ were added in the spinning dope to be embedded within the generated nanofibers. These nanoparticles show certain optical activity due to optical trivaliant cerium ions, associated with formed oxygen vacancies. The formed nanocomposite shows promising mechanical properties such as the Young's modulus, elasticity (or elongation at break), and toughness. In addition, the electrospun mat becomes fluorescent with 520-nm emission upon exposure to UV light, due to excitation of the optically active ceria nanoparticles. Also, the formed nanocomposite shows a decay of its electric resistance over time upon exposure to cyclic loads at different humidity conditions. The synthesized nanocomposite can be utilized in different biomedical, textile, and sensing applications. (c) 2018 Society of PhotoOptical Instrumentation Engineers (SPIE) [DOI: [0.1117/1].JNPI12.026016]
\end{abstract}

Keywords: spider silk; electrospinning; nanofibers; ceria nanoparticles; fluorescence.

Paper 18005P received Jan. 22, 2018; accepted for publication May 31, 2018; published online Jun. 13, 2018.

\section{Introduction}

Spider silks (SS) are considered the strongest, most elastic, and toughest biomanufactured fibers. Web-weaving spiders have used impressive silks where the proteins in the silks can be described as nondiverged at the protein sequence level ${ }^{\text {喵 }}$ In the last three decades, several research papers have studied the physical, mechanical, and chemical properties of SSs, as promising biomaterials for a variety of applications including neural system regrowth and other applications such as textile ${ }^{\mathrm{B}}$ However, the study of their optical and electrical properties is still lacking for the most part. The mechanical and frictional forces align the protein molecules to form the fibers ranging from 20 to under $1 \mu \mathrm{m}$ in diameter. Mechanical properties of natural SSs are promising due to its ability to absorb the energy of the prey without damaging of the web, in addition to avoiding any rebound of the insect away from the web. ${ }^{\mathbb{Q}}$ Different parameters affect the properties of the protein silk fiber such as temperature, hydration state, protein concentration, and extension rate. Major ambulate silk (MaSp) is a two protein-composition in every orb-weaving SS ${ }^{⿴ 囗 十}$ MaSp proteins are composed of GGX, GPGXX, and poly-alanine repeats, where X could

*Address all correspondence to: Ishac Kandas, E-mail: Ukandas@kcstedukw

1934-2608/2018/\$25.00 (C) 2018 SPIE 
be glutamine $(\mathrm{Q})$, tyrosine $(\mathrm{Y})$, or leucine $(\mathrm{L})$. Recent research also revealed a hierarchical structure of the silk fibers that led to a better understanding of their promising mechanical performance 매밀 However, very little research has been done on the formation of nanofibers recombinant spider silk protein (rSSPs) via electrospinning process with both electrical and optical characterizations [1] [1]

Our work suggests electrospinning of rSSP (MaSp1) nanofibers with active cerium oxide nanoparticle (ceria NPs) to create an electrospun mat with specific optical characteristics can be possibly used for biomedical applications. Ceria NPs have a strong capability to act as free radical scavenger and to capture dissolved oxygen in liquid media. ${ }^{[5}$ These characteristics allow ceria NPs to find their possible applications in cancer treatment, energy applications (solar cells), or detection tools (e.g., oxygen and peroxide sensors). 10 Cerium ions could exist in either an active trivalent state $\left(\mathrm{Ce}^{3+}\right)$ with associated charged oxygen vacancies or nonactive $\left(\mathrm{Ce}^{4+}\right)$ with no formed $\mathrm{O}$-vacancies, with a major possibility of having mixed ionization states as a nonstoichiometric compound $\mathrm{CeO}_{2-x}$. The trivalent cerium ions show fluorescence characteristics when are exposed to UV light. ${ }^{20}$

Ceria nanoparticles are prepared using a simple chemical precipitation technique. ${ }^{\text {T }}$ Then, the embedded ceria nanoparticles are added in situ with SS solution and electrospun into nanofibers. In this work, the embedded ceria nanoparticles are shown to add new optical feature for the spider silk nanofibers (SS NFs), fluorescence, in addition to data on both the mechanical and electrical characterization of the formed nanocomposite.

\section{Experimental Work}

\subsection{Materials}

The recombinant spider silk dragline protein (MaSp1) was purified (90+\% pure) from the milk of genetically modified goats based on the purification method mentioned in Ref. 22 and 23 . The purified MaSp1 protein was found to consists of two major amino acid sequence: (1) GGA-GGX $[\mathrm{X}=$ tyrosine $(\mathrm{Y})$, glutamine $(\mathrm{Q})$, and lysine $(\mathrm{L})]$, which are acting as soft flexible segments and (2) alanine blocks (AAAAAAAAAA), each block consists of 8 to 10 alanine monomeric unit forming B-sheets, which are acting as strength element in the drag line SS. The whole sequence is GGAGQGGYGGQGAGQGGYGGLGSQGAGRGGLGGQGAGAAAAAAAA and repeated for 16 times to form a giant polyamide chain with the average molecular weight of 60 to $65 \mathrm{kDa}$.

Formic acid (88\%) was purchased from Alfa Aesar (Ward Hill, Massachusetts) and 1,1,1,3,3,3-hexa-fluoro 2-isopropanol (HFIP) was purchased from Oakwood chemicals (Estill, South Carolina). Triton X-100 from Sigma-Aldrich (St. Louis, Missouri) has been used as surfactant. Cerium (III) chloride heptahydrate was purchased from Sigma-Aldrich (St. Louis, Missouri) and used as precursor for ceria NPs. Ammonium hydroxide and ethanol were used during synthesis of ceria Nps.

\subsection{Synthesis of Ceria NPS}

Ceria nanoparticles are first synthesized using a chemical precipitation technique similar to Chen et al. but with some modifications. ${ }^{-4}$ Synthesis of cerium oxide nanoparticles as follows. Cerium (III) chloride heptahydrate ( $0.5 \mathrm{~g}$, Sigma-Aldrich) is inserted into a beaker with adding $40 \mathrm{~mL}$ of distilled water, and the solution is stirred using a magnetic stirrer at rate of $500 \mathrm{rpm}$ for $24 \mathrm{~h}$. The solution is heated to $50^{\circ} \mathrm{C}$ in a hot water bath for $2 \mathrm{~h}$ with the addition of $1.6 \mathrm{~mL}$ of commercial ammonium hydroxide. Then, it is stirred for $22 \mathrm{~h}$ at room temperature. The long period of stirring fractures any remaining nanorods into nanoparticles. The solution is then centrifuged, washed with deionized water and ethyl alcohol.

\subsection{Electrospinning Dope Preparation}

The SS solution was initially prepared by dissolving MaSp1 protein in a mixture of formic acid of $88 \%$ and HFIP at a ratio of 1:1. $0.1 \mathrm{~mL}$ of Triton X-100 was added as a surfactant. The mixture 


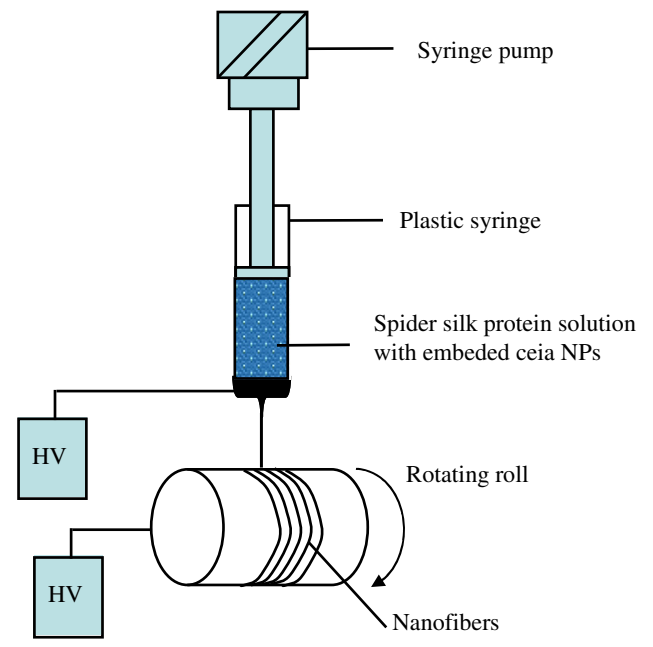

Fig. 1 Simple schematic diagram of the electrospinning setup.

was sonicated using an ultrasonication probe (Fischer, $1 \mathrm{~W}$ ) for $3 \mathrm{~min}$ prior to the addition of ceria nanoparticles. The diluted ceria nanoparticles dispersion $(0.02 \mathrm{~g} / \mathrm{L})$ was added to the SS solution and stirred for few minutes using vortex mixer to form the final spinning solution.

\subsection{Electrospinning Process}

The electrospinning unit purchased from IME Technologies (Geldrop, The Netherlands), consists of a high voltage power supply and a syringe pump, which is used to regulate the pumping rate of the polymer solution, a $5-\mathrm{mL}$ plastic syringe with 30 -gauge metallic needle, and a rotating metallic collector of radius $10 \mathrm{~cm}$ covered with nonsticky aluminum foil was used as a target. A schematic illustration of the electrospinning setup is shown in Fig. [1. The voltage power supply is connected to the needle while the collector is grounded with a separation distance of $15 \mathrm{~cm}$. The voltage difference between the needle and target is $26 \mathrm{kV}$, with a flow rate of the polymer solution at $1 \mathrm{~mL} / \mathrm{h}$ for $1 \mathrm{~h}$ running time per sample. The speed of rolling collector is set to $1500 \mathrm{rpm}$.

\section{Characterization}

\subsection{Scanning Electron Microscopy}

The electrospun fibers were characterized by field emission scanning electron microscopy (FEI Quanta 200, Hillsboro, Oregon) to characterize their morphology and fiber diameter. The electrospun mats were mounted on an aluminum stub and coated with a gold layer about 10-nm thick. The mean fiber diameter was calculated as an average of 200 measurements using Image $\mathbf{J}$ software.

\subsection{Transition Electron Microscopy}

The mean synthesized nanoparticle size was observed by transmission electron microscopy (TEM, JEOL, Tokyo, Japan), with accelerating potential of $80 \mathrm{kV}$.

\subsection{Mechanical Properties Measurements}

The mechanical properties of the yarns were tested using an MTS tensile tester (Synergy 100, MTS, Eden Prairie, Minnesota) with custom 10-g load cell (Transducer Technique). The electrospun mats were cut longitudinally into strips of size $1 \times 12 \mathrm{~cm}$. Each strip was twisted using a fringe twister (Lacis cord maker and fringe twister, Berkeley, California) for $10 \mathrm{~s}$ into yarns of 
average diameters 300 to $700 \mu \mathrm{m}$. The twisted yarns were then fixed on C-shaped plastic holders with a fixed gap between holder's tips of $19.1 \mathrm{~mm}$ and secured in the tensile testing instrument. The tensile test was run at a strain rate of $5 \mathrm{~mm} / \mathrm{min}$ and the average of 10 individual measurements was calculated.

\subsection{Optical Activity Measurement}

Spider silk protein nanofibers (rSSP NFs) with and without embedded ceria NPs were optically characterized by measuring absorbance and fluorescence intensity curves. Optical absorbance from 350 to $550 \mathrm{~nm}$ was measured using ultraviolet-visible (UV-vis) spectrophotometer (PG T92+, Beijing, China). From absorbance curves, the corresponding band gap of the formed nanocomposite can be determined, as discussed below. The fluorescence setup is composed of a violet light-emitting diode (LED) from Thorlab (Newton, New Jersey) with a 430-nm excitation wavelength, a monochromator (Newport Cornerstone 130, Irvine, California), which was set to obtain fluorescence intensity at wavelengths from 500 to $700 \mathrm{~nm}$, an Oriel photomultiplier tube (PMT) (Newport PMT77340, Newport, Irvine, California) as a fluorescence intensity detector, and a power meter (Newport 1918-R, Newport, Irvine, California) to display PMT detection readings. Fluorescence intensity was measured by positioning the NFs solid sample holder between the UV-LED and the input port of the monochromator, so the input optical signal to the monochromator is perpendicular to the initial LED excitation signal for minimum scattering effect. ${ }^{27}$ In other words, the fluorescent signal is to be inclined by $90 \mathrm{deg}$ compared to the incident light for minimum scattering effect. The output port of the monochromator was directly connected with PMT, which was directly connected to 1918-R power meter.

\subsection{Electrical Properties}

The electrical properties of the designed nanocomposite have been determined through a simple setup as shown in Fig. 2. The nanocomposite was twisted into yarns of diameters 500 to $700 \mu \mathrm{m}$ with a length of $\sim 4 \mathrm{~cm}$. Each end of the yarn is attached to a metal clamp connected to a commercial multimeter. The controlled environment is then set to approximated relative humidity (RH) level and the SS's resistance is recorded as a function of time and humidity. The resistance value was generally recorded for $\sim 30$ min per experiment. A RH level of $99 \%$ is reached to offer a fully humid environment for the prepared nanocomposite.

\section{Results}

\subsection{Spider Silk Nanofiber and Cerium Oxide Morphology}

SEM image of the electrospun nanofibers in Fig. 3 shows that the fiber diameters are ranged around $50 \pm 20 \mathrm{~nm}$. With the assumption that the air gaps between the individual fibers are neglected, the approximate number of fibers contributed in yarn formation ranged from

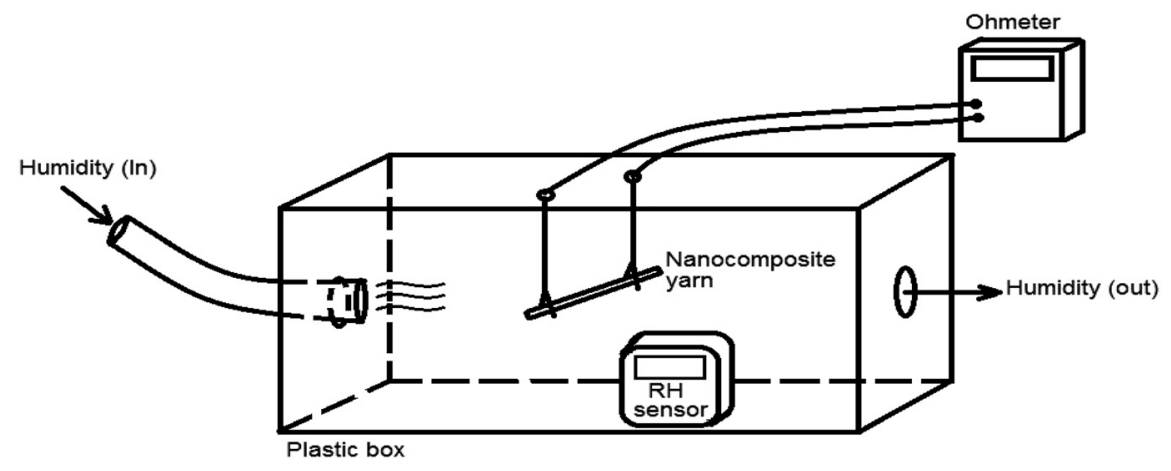

Fig. 2 Schematic diagram of resistance measurement setup. 
(a)

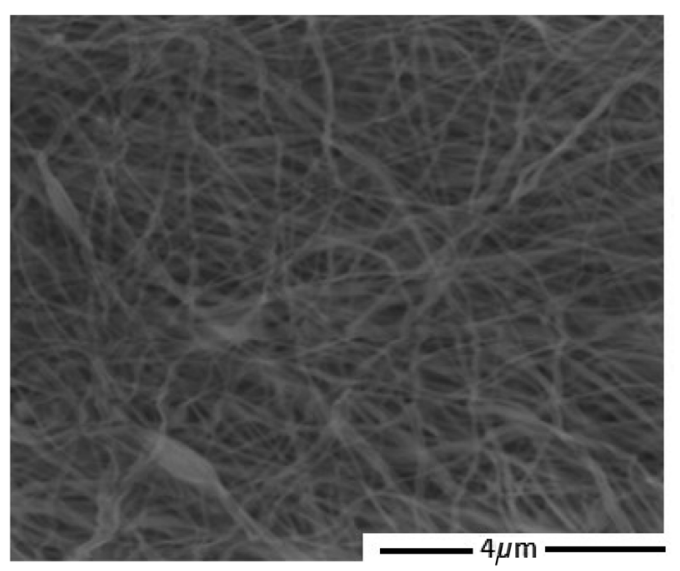

(b)

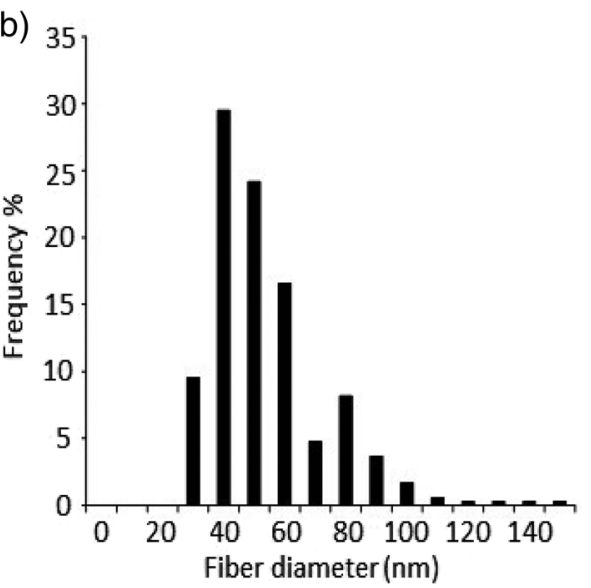

Fig. 3 (a) SEM image of the electrospun SS NPs and (b) the fiber diameter distribution (the fiber diameters in the range of $50 \pm 20 \mathrm{~nm}$ ).

6000 to 14,000 aligned nanofibers. Upon addition of cerium oxide nanoparticles, no significant changes have been observed in the fiber diameters.

A TEM image of freshly prepared ceria nanoparticles is shown in Fig. 4(a), with mean diameter of $6 \mathrm{~nm}$. Another TEM image of aggregated ceria nanoparticles inside the nanofibers surface is shown in Fig. 4(b), It is not recommended to add any surfactant to reduce the nanoparticles aggregation, because it can reduce the formed $\mathrm{O}$-vacancies inside ceria crystalline structure that can affect the optical properties of ceria nanoparticles and the entire nanocomposite negatively. ${ }^{5}$

\subsection{Optical Properties}

The absorbance curves of the SS NPs with embedded ceria NPs are shown in Fig. 5(a). The absorbance increases within shifting to lower wavelength, which is correlated to the embedded ceria NPs. The optical allowed direct bandgap can be calculated directly from the obtained absorbance curves using

$$
\alpha E=A\left(E-E_{g}\right)^{1 / 2}
$$

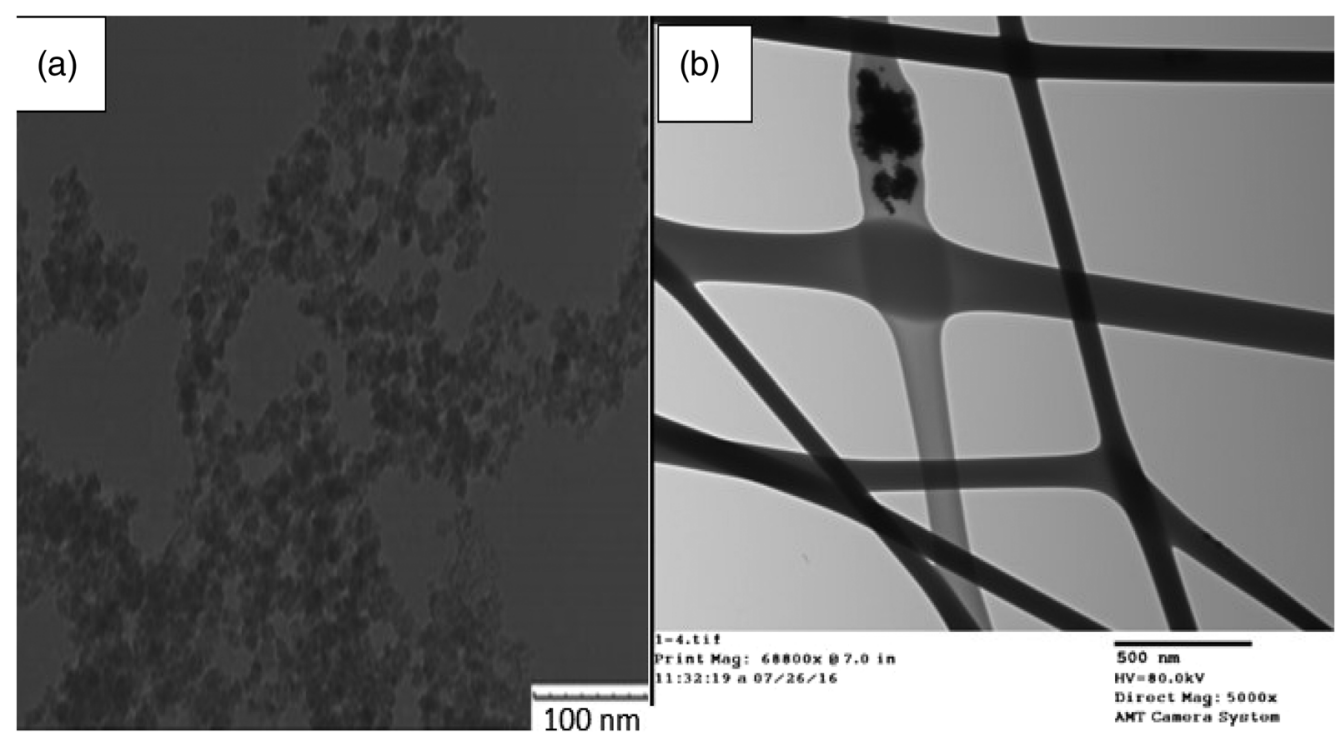

Fig. 4 (a) TEM image for the prepared cerium oxide nanoparticles and (b) aggregated nanoparticles inside the electrospun nanofibers. 

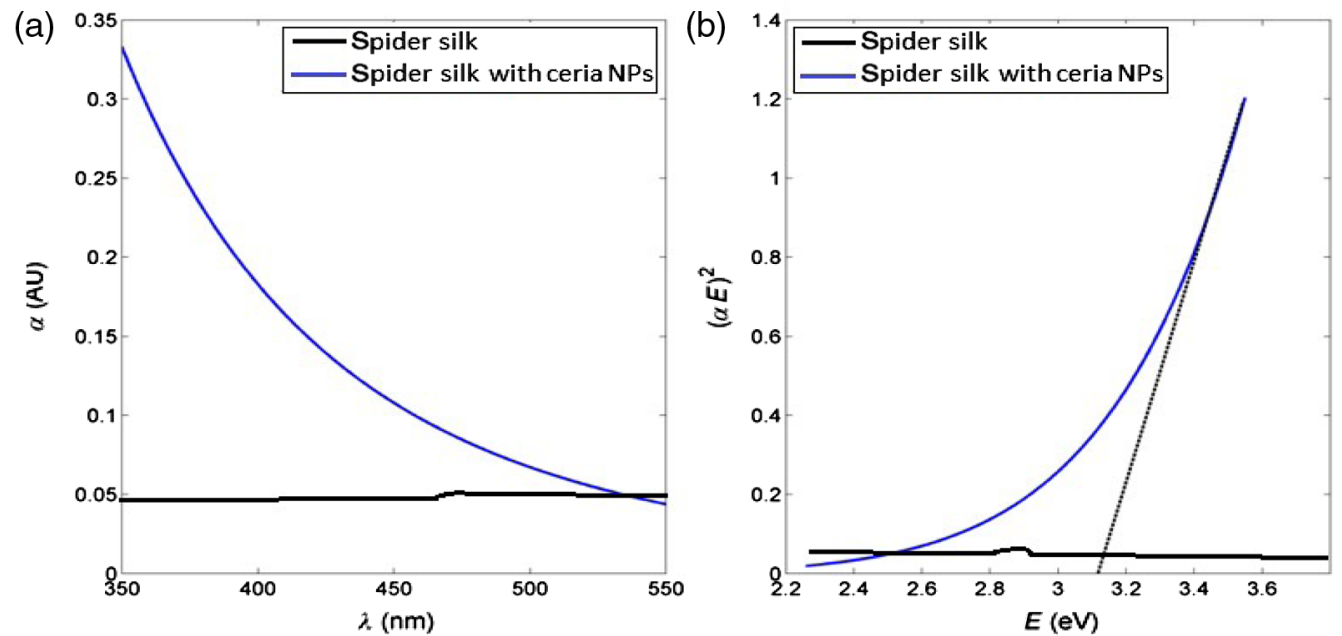

Fig. 5 (a) Absorbance dispersion and (b) bandgap calculations of SS NPs with/without embedded ceria nanoparticles.

where $\alpha$ is the absorbance coefficient, $A$ is the material constant that depends on the effective masses of electrons and holes in the ceria NPs, $E$ is the absorbed photon energy, and $E_{g}$ is the allowed direct bandgap.

Figure 5(b) shows the relation between $(\alpha E)^{2}$ versus $E$, and the intersection of the extrapolation of the linear part of the $(\alpha E)^{2}$ curve with the $E$-axis is equal to the allowed direct bandgap $E_{g}$. The bandgap is found to be $<3.2 \mathrm{eV}$, which confirms that cerium ions have a dominant concentration of trivalent states associated with oxygen vacancies, which can be responsible for the fluorescence properties of ceria nanoparticles and the whole nanocomposite when embedding such nanoparticles inside SS NPs. 20

Fluorescence intensity measurements are shown in Fig. 6 of ceria NPs which were embedded in situ in SS NPs. The fluorescence emission appears at a wavelength close to $500 \mathrm{~nm}$ under 430nm excitation, which is one of the embedded ceria NPs' optical characteristics, with corresponding electron transitions of $5 \mathrm{~d}-4 \mathrm{f}$ molecular levels inside the cerium trivalent ionization states, with no fluorescence emission from the pure SS NPs.7 Comparing to crosslinked PVA nanofibers as cited in Ref. 28, it is observed that the obtained bandgap of ceria becomes lower in case of SS compared to crosslinked PVA host. Also, the fluorescence intensity amplitude is higher in case of SS host compared to crosslinked PVA. That can be explained as the crosslinking process may affect the activity of trivalent cerium ions and the corresponding O-vacancies. That makes the SS host better in keeping the activity of trivalent cerium ions with better optical characteristics.

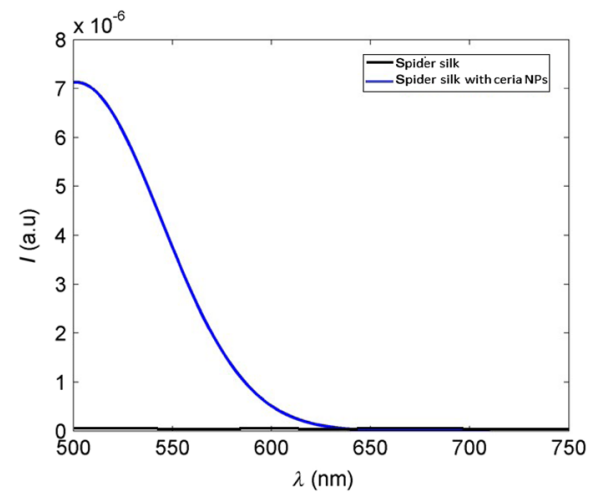

Fig. 6 Fluorescence intensity of SS NFs with in situ embedded with ceria NPs. 


\subsection{Mechanical Properties}

Regarding the mechanical properties, Fig. 7 shows the mechanical properties of SS NPs with and without adding ceria nanoparticles at normal RH (16\%). As shown in Fig. 7, all the mechanical properties such as elastic modulus, tensile strength, maximum strain, strength at break, and energy of break are reduced when ceria NPs are added. Table 11 summarizes the comparison between SS only and SS with added ceria NPs. However, this difference can be considered a tradeoff compared to both optical and electrical benefits from adding ceria NPs. Figure 8 shows stress-strain analysis of SS NPs nanofibers with embedded ceria NPs at normal RH $(\mathrm{RH}=16 \%)$ and fully humid surrounding medium $(\mathrm{RH} \sim 99 \%)$. The number of samples was about five and the average curve is shown in Fig. 7. The standard deviation is also shown in the table. As seen from Table \& the elastic modulus is reduced $\sim 50 \%$. Also, the tensile strength and strength at break (at the elastic limit) are reduced as well. The maximum strain is

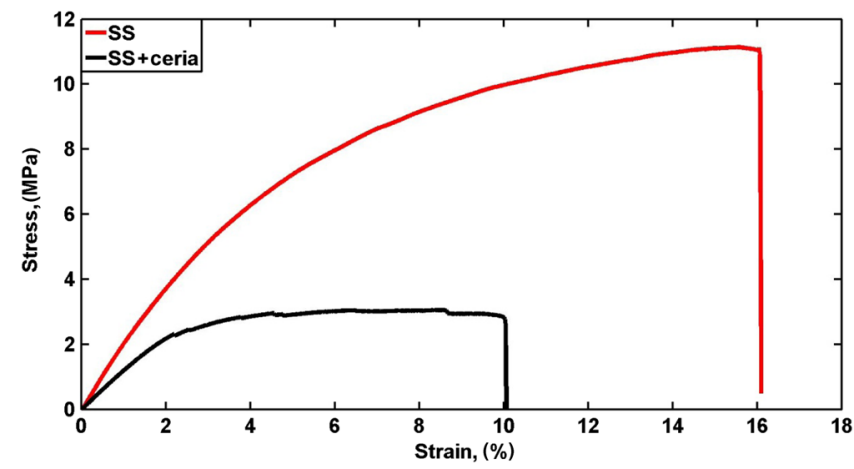

Fig. 7 Stress-strain mean curve for SS nanofibers and SS nanofibers embedded with ceria NPS.

Table 1 Mean values of mechanical properties comparison between SS NFs and SS NFs embedded with ceria NPs.

\begin{tabular}{lcc}
\hline \hline & SS NFs & SS NFs with ceria NPs \\
\hline Elastic modulus (MPa) & $1.89 \pm 0.31$ & $1.14 \pm 0.17$ \\
Tensile strength (MPa) & $3.57 \pm 0.50$ & $1.96 \pm 0.44$ \\
Maximum strain (\%) & $16.09 \pm 4.2$ & $10.06 \pm 2.51$ \\
Strength at break (MPa) & $11.13 \pm 1.88$ & $3.05 \pm 0.56$ \\
Energy to break $\left(\mathrm{kJ} / \mathrm{m}^{2}\right)$ & $24.67 \pm 7.97$ & $4.84 \pm 1.49$ \\
\hline \hline
\end{tabular}

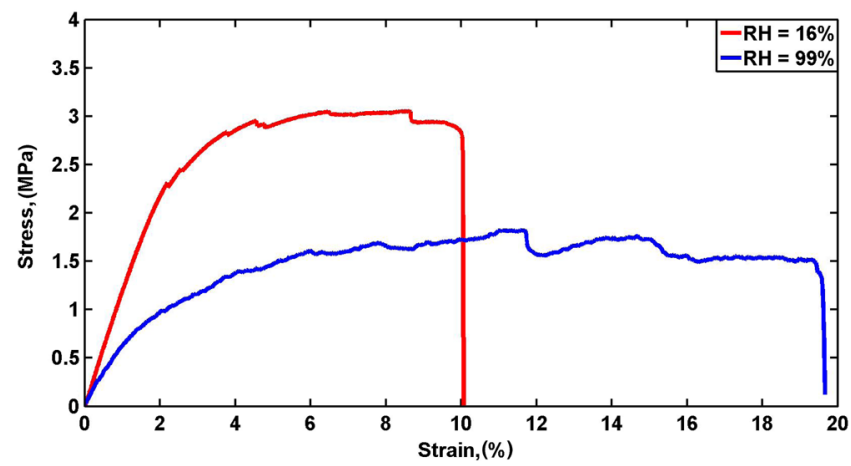

Fig. 8 Stress-strain mean curve for nanocomposite of SS NFs embedded with ceria NPs at different $\mathrm{RH}$ values. 
Table 2 Mean values of mechanical properties comparison between SS nanofibers embedded with ceria NPs at different RH values.

\begin{tabular}{lcr}
\hline \hline & Normal RH (RH =16\%) & $\mathrm{RH}=99 \%$ \\
\hline Elastic modulus (MPa) & $1.14 \pm 0.17$ & $0.58 \pm 1.01$ \\
Tensile strength (MPa) & $1.96 \pm 0.44$ & $0.69 \pm 0.49$ \\
Maximum strain (\%) & $10.06 \pm 2.51$ & $19.66 \pm 8.32$ \\
Strength at break (MPa) & $3.05 \pm 0.56$ & $1.82 \pm 0.67$ \\
Energy to break $\left(\mathrm{kJ} / \mathrm{m}^{2}\right)$ & $4.84 \pm 1.49$ & $5.45 \pm 2.72$ \\
\hline \hline
\end{tabular}

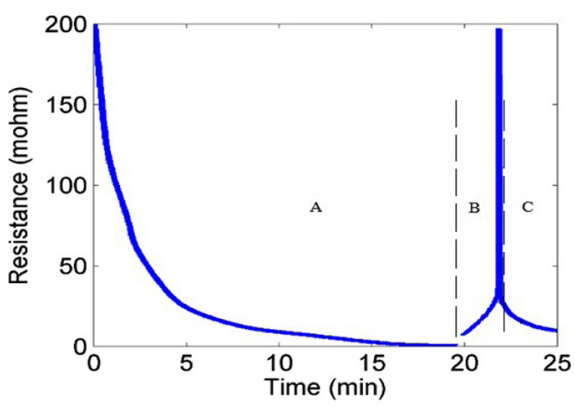

Fig. 9 Cyclic resistance of the nanocomposite; SS with ceria NPs, at RH levels of $99 \%$ and $16 \%$ "normal condition of the lab" (section A: resistance decay over time at $\mathrm{RH}=99 \%$, section $\mathrm{B}$ : rapid increase of resistance at normal lab environment at $\mathrm{RH}=16 \%$, and section $\mathrm{C}$ : cyclic return of resistance decay over another full humid environment at $\mathrm{RH}=99 \%$ ).

approximately doubled, and the energy of break (area under the curve) is increased a small amount. For each measurement, five samples are used, then, the mean values and standard deviations are calculated.

\subsection{Electrical Properties}

For the electric resistance measurement in the case of $\mathrm{RH} \sim 99 \%$, as shown in Fig. Q 7 in section A, the resistance values are observed as exponential-like decay for the SS NPs with increasing the exposure time to the humid environment. This may be due to the shrinkage of the SS proteins. In more detail, as the $\mathrm{RH}$ reduced down to $16 \%$, the resistance elevates by the trend shown in Fig. 9 . When the RH returned back to the 99\%, the resistance drops again. The shrinkage of the SS yarns could be due to distortion of the fibroin chains by water, which were aligned along the nanofibers axis. The required time for a significant drop of the measured resistance was found to be relatively high, up to $20 \mathrm{~min}$ at the saturation level. However, in normal RH levels, the resistance starts to increase substantially with relatively short rise time. The resistance alteration at different $\mathrm{RH}$ values was shown to be cyclic during the variation of $\mathrm{RH}$ due to a cyclic shrinkage nature of silk spider proteins with approximately no change in the macroscopic state of the yarn itself. The diameter of the yarn is reduced up to $50 \%$ of its original diameter during the first 1 to 2 min at RH 99\%, and then the diameter stays constant. The length of the yarn is found to be approximately constant. That can give an explanation of the cyclic effect of SS/ceria nanofibers is due to mainly the cyclic shrinkage/extension of the SS proteins rather than a change in the outer dimensions of the yarns.

\section{Conclusions}

The work demonstrates the acquiring electrospun SS NPs for fluorescence through embedding ceria nanoparticles. Ceria nanoparticles with a largely $\mathrm{Ce}^{3+}$ ionization state have been added 
in situ to MaSp1 SS protein solution and then electrospun into nanofibers. The average electrospun fiber diameter is around $50 \mathrm{~nm}$ with embedded ceria nanoparticles of mean size around $6 \mathrm{~nm}$. The nanocomposite shows fluorescence characteristic with visible emission under UV excitation and with direct optical bandgap around $3.2 \mathrm{eV}$. The manufactured nanocomposite shows a maximum strain up to $20 \%$. The electric resistance of the nanocomposite is found to decay with time in humid environment. The increase and decrease in nanocomposite electrical resistance show a kind of cyclic behavior when the humidity is changed. So, our contribution is to fabricate multifunctional nanocomposite with having different characteristics including biocompatibility, optical fluorescent, and quite electrical conductive in humid environment. This work can be helpful in applying the electrospun SS NPs in biomedical applications due to its small diameters, biocompatibility, and the ability to implement different materials to impart different functionalities. Also, the synthesized nanocomposite using ceria NPs could be very useful in cancer treatment due to its properties of being free radical scavenger and capturing dissolved oxygen in liquid media.

\section{Acknowledgments}

This work was made possible by NPRP Grant No. NPRP 7-1724-3-438 from the Qatar National Research Fund (a member of the Qatar Foundation). The statements made herein are solely the responsibility of the authors.

\section{References}

1. F. Lucas, "Spiders and their silks," Discovery 25, 20-26 (1964).

2. F. Vollrath, "Spider webs and silks," Sci. Am. 266, $70-76$ (1992).

3. C. Copeland et al., "About development of a process for the spinning of synthetic spider silk," ACS Biomater. Sci. Eng. 1, 577-584 (2015).

4. P. J. Willcox et al., "Evidence of a cholesteric liquid crystalline phase in natural silk spinning processes," Macromolecules 29, 5106-5110 (1996).

5. F. Vollrath and D. P. Knight, "Liquid crystalline spinning of spider silk," Nature 410, 541548 (2001).

6. L. Zhou et al., "Naturally derived carbon nanofibers as sustainable electrocatalysts for microbial energy harvesting: a new application of spider silk," Appl. Catal. B 188, 31-38 (2016).

7. S. Stauffer, S. Cougill, and R. V. Lewis, "Comparison of physical properties of three silks from Nephila clavipes and Araneus gemmoides," J. Arachnol. 22, 5-11 (1994).

8. E. Fischer, "About spider silk, Hoppe-Seyler's," T. Physiol. Chem. 53, 126-139 (1907).

9. M. Xu and R. V. P. Lewis, "Structure of a protein superfiber: spider dragline silk," Proc. Natl. Acad. Sci. U. S. A. 87, 7120-7124 (1990).

10. M. B. Hinman and R. V. Lewis, "Isolation of a clone encoding a second dragline silk fibroin,” J. Biol. Chem. 267, 19320-19324 (1992).

11. H.-J. Jin and D. Kaplan, "Mechanism of silk processing in insects and spiders," Vature 424, 1057-1061 (2003).

12. Q. Yu et al., "Structure-property relationship of regenerated spider silk protein nano/microfibrous scaffold fabricated by electrospinning," t. Biomed. Mater. Res. A 102, 3828-3837 (2014).

13. A. Steins et al., "In vitro evaluation of spider silk meshes as a potential biomaterial for bladder reconstruction," PLoS One 10, e0145240 (2015).

14. S. Keten et al., "Nanoconfinement controls stiffness, strength and mechanical toughness of beta-sheet crystals in silk," Nat. Mater. 9, 359-367 (2010).

15. D. Porter, F. Vollrath, and Z. Shao, "Predicting the mechanical properties of spider silk as a model nanostructured polymer," Eur. Phys. J. E. Soft. Matter. 16, 199-206 (2005).

16. M. Quaranta, S. M. Borisov, and I. Klimant, "Indicators for optical oxygen sensors," Bioanal. Rev. 4, 115-157 (2012). 
17. N. Feng, J. Xie, and D. Zhang, "Synthesis, characterization, photophysical and oxygensensing properties of a novel europium(III) complex," Spectrochim. Acta A Mol. Biomol. Spectrosc. 77, 292-296 (2010).

18. B. A. Rzigalinski et al., "Radical nanomedicine," Nanomedicine 1, 399-412 (2006).

19. N. Shehata et al., "Nano-enriched and autonomous sensing framework for dissolved oxygen," Sensors 15, 20193-20203 (2015).

20. N. Shehata et al., "Enhanced erbium-doped ceria nanostructure coating to improve solar cell performance," Materials 8, 7663-7672 (2015).

21. N. Shehata et al., "Control of oxygen vacancies and $\mathrm{Ce}^{+3}$ concentrations in doped ceria nanoparticles via the selection of lanthanide element," T. Nanopart. Res. 14, 1173-1183 (2012).

22. C. Tucker et al., "Mechanical and physical properties of recombinant spider silk films using organic and aqueous solvents," Biomacromolecules 15, 3158-3170 (2014).

23. J. Jones et al., "More than just fibers: an aqueous method for the production of innovative recombinant spider silk protein materials," Biomacromolecules 16, 1418-1425 (2015).

24. H. Chen and H. Chang, "Homogeneous precipitation of cerium dioxide nanoparticles in alcohol/water mixed solvents," Colloids Surf. A 242, 61-69 (2004).

25. N. Shehata, "Design of optical characteristics of ceria nanoparticles for applications including gas sensing and up-conversion," PhD Thesis, Virginia Tech University (2012).

26. J. I. Pankove, Optical Processes in Semiconductors, Prentice-Hall, Englewood Cliffs, New Jersey (1971).

27. P. Patsalas et al., "Structure-dependent electronic properties of nanocrystalline cerium oxide films," Phys. Rev. B 68, 035104 (2003).

28. N. Shehata et al., "Fluorescent nanocomposite of embedded ceria nanoparticles in crosslinked PVA electrospun nanofibers," Nanomaterials 6, 102 (2016).

Biographies for the authors are not available. 